\title{
ON $Y$-COORDINATES OF PELL EQUATIONS WHICH ARE BASE 2 REP-DIGITS
}

\author{
Bernadette Faye-Fall and Florian Luca \\ Université Gaston Berger, Sénégal and University of the Witwatersrand, \\ South Africa
}

\begin{abstract}
In this paper, we show that if $\left(X_{k}, Y_{k}\right)$ is the $k$ th solution of the Pell equation $X^{2}-d Y^{2}=1$ for some non-square integer $d>1$, then the equation $Y_{k}=2^{n}-1$ has at most two positive integer solutions $(k, n)$.
\end{abstract}

\section{INTRODUCTION}

Let $d>1$ be an integer which is not a square. The Pell equation

$$
X^{2}-d Y^{2}=1
$$

has infinitely many positive integer solutions $(X, Y)$. Furthermore, putting $\left(X_{1}, Y_{1}\right)$ for the smallest such (i.e., the solution with the smallest value of $\left.Y\right)$, all other solutions are of the form $\left(X_{k}, Y_{k}\right)$ where

$$
X_{k}+\sqrt{d} Y_{k}=\left(X_{1}+\sqrt{d} Y_{1}\right)^{k} \quad \text { for all } \quad k \geq 1 .
$$

Let $U$ be some interesting set of positive integers like squares, rep-digits in base 10 or in an arbitrary base $g>1$, Fibonacci numbers, Tribonacci numbers, factorials, etc. In recent papers the question of determining all positive integers $d$ such that $X_{k} \in U$ holds for at least two positive integers $k$ has been investigated (see $[3,4,6,7,9,10])$. In all cases mentioned above, there are only finitely many such $d$, meaning that with these finitely many exceptions in $d$, the equation $X^{2}-d Y^{2}=1$ has at most one positive integer solution $(X, Y)$ with $X \in U$. That this is best possible follows from the fact that if $u \in U \backslash\{1\}$, then $(X, Y)=(u, 1)$ is a solution to $X^{2}-d Y^{2}=1$ for $d:=u^{2}-1$.

In this paper, we start the same program for the coordinate $Y$. Here, it is easy to construct infinitely many $d$ such that $Y_{k} \in U$ has two solutions $k$.

2020 Mathematics Subject Classification. 11B39, 11D61.

Key words and phrases. Pell equations, exponential Diophantine equations, applications of linear forms in logarithms. 
Namely, assume that $1 \in U$. Take $d=u^{2}-1$, where $u$ will be determined later. Then $\left(X_{1}, Y_{1}\right)=(u, 1)$ and $\left(X_{2}, Y_{2}\right)=\left(2 X_{1}^{2}-1,2 X_{1} Y_{1}\right)=\left(2 u^{2}-1,2 u\right)$. Hence, if also $2 u \in U$, then for this $d$, we have $Y_{k} \in U$ for both $k=1,2$. Thus, if $U$ contains 1 and infinitely many even numbers, then there are infinitely many $d$ such that $Y_{k} \in U$ for both $k=1,2$. We ask if this is best possible, meaning whether for particular interesting sets of positive integers $U$, the containment $Y_{k} \in U$ holds for 3 or more values of $k$ only for a finite set of $d$. We mention that the question of how many solutions $k$ does $Y_{k} \in U$ have has been studied before for a couple of interesting sets $U$. For example, if $U$ is the set of squares, then Ljunggren ([8]) showed that there are at most two such $k$. Further, if $U$ is the set of $Y$-coordinates of a Pell equation corresponding to the non-square integer $d_{1}>1$, then for any non-square positive integer $d \neq d_{1}$, the containment $Y_{k} \in U$ has at most three solutions $k$. This is a result of Bennett ([2]) which improved upon a prior result of Masser and Rickert ([11]) who had shown an upper bound of at most 16 on the number of such solutions $k$.

In this paper, we take $U:=\left\{2^{n}-1: n \geq 1\right\}$ which is the set of rep-digits in base 2 . We prove the following theorem.

THEOREM 1.1. Let $d>1$ be an integer which is not a square and let $\left(X_{k}, Y_{k}\right)$ be the sequence of positive integer solutions to $X^{2}-d Y^{2}=1$. Then the equation $Y_{k}=2^{n}-1$ has at most two positive integer solutions $(k, n)$.

The conclusion of Theorem 1.1 is best possible. To see this we take $d=2^{2 \ell}-1$ for some positive integer $\ell$. For such $d$ we have $\left(X_{1}, Y_{1}\right)=\left(2^{\ell}, 1\right)$ and $\left(X_{3}, Y_{3}\right)=\left(2^{3 \ell+2}-3 \cdot 2^{\ell}, 2^{2 \ell+2}-1\right)$, so the equation $Y_{k}=2^{n}-1$ has the solutions $(k, n)=(1,1),(3,2 \ell+2)$. We leave it to the reader to determine if these are the only $d$ such that the equation $Y_{k}=2^{n}-1$ has two solutions $(k, n)$, or whether there are other values of $d$ with the above property.

The method of proof follows the approach from [5].

\section{Preliminaries on Pell equations}

In this section, we collect some useful facts about Pell equations. Let

$$
\alpha:=X_{1}+\sqrt{d} Y_{1} \quad \text { and } \quad \beta:=X_{1}-\sqrt{d} Y_{1}=\alpha^{-1} .
$$

Since $X_{1}, Y_{1}, d>1$ are all positive integers, it follows that $\alpha>1+\sqrt{2}$. Then

$$
X_{k}=\frac{\alpha^{k}+\beta^{k}}{2} \quad \text { and } \quad Y_{k}=\frac{\alpha^{k}-\beta^{k}}{2 \sqrt{d}} \quad \text { hold for all } \quad k \geq 1 .
$$

In particular,

$Y_{k}=\frac{\alpha^{k}-\beta^{k}}{2 \sqrt{d}} \geq \frac{\alpha^{k}-\beta^{k}}{2 \sqrt{d} Y_{1}}=\frac{\alpha^{k}-\beta^{k}}{\alpha-\beta}=\alpha^{k-1}+\alpha^{k-2} \beta+\cdots+\beta^{k-1} \geq \alpha^{k-1}$. 
The above inequality $Y_{k} \geq \alpha^{k-1}$ is strict for $k>1$. There are many formulas relating members of the sequence $\left\{X_{k}\right\}_{k \geq 1}$ and $\left\{Y_{k}\right\}_{k \geq 1}$. For example,

$$
X_{2 k}=2 X_{k}^{2}-1 \text { and } Y_{2 k}=2 X_{k} Y_{k} \text { hold for all } k \geq 1 .
$$

Furthermore, if $k>\ell$, then

$$
\begin{aligned}
& Y_{k}-Y_{\ell}=2 Y_{(k-\ell) / 2} X_{(k+\ell) / 2} \\
& Y_{k}+Y_{\ell}=2 Y_{(k+\ell) / 2} X_{(k-\ell) / 2}
\end{aligned} \quad \text { hold provided } k \equiv \ell \quad(\bmod 2) .
$$

Moreover, if $\ell \mid k$, then $Y_{\ell} \mid Y_{k}$. If in addition $k / \ell$ is odd, then also $X_{\ell} \mid X_{k}$. We will need all these facts later. The following result is well-known (see [5]). In what follows, for a nonzero integer $a$ we write $\nu_{2}(a)$ for the exponent of 2 in the factorisation of $a$.

LEMMA 2.1. We have:

(i)

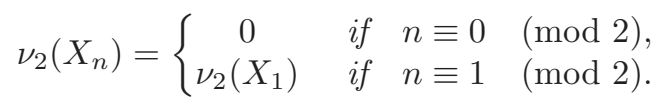

(ii) Let $e$ be the smallest of positive integer $k$ such that $2 \mid Y_{k}$. Then $e \in\{1,2\}$. Furthermore, $\nu_{2}\left(Y_{n}\right)=0$ except if $e \mid n$ in which case

$$
\nu_{2}\left(Y_{n}\right)=\nu_{2}\left(Y_{e}\right)+\nu_{2}(n / e) .
$$

\section{The PROOF OF THE THEOREM}

We briefly discuss the strategy of the proof. From now on until the end of the paper we assume that $\left(k_{1}, n_{1}\right),\left(k_{2}, n_{2}\right),\left(k_{3}, n_{3}\right)$ are solutions $(k, n)$ to $Y_{k}=2^{n}-1$ with $k_{1}<k_{2}<k_{3}$. Hence, $n_{1}<n_{2}<n_{3}$. Since $Y_{1} \mid Y_{k}$ for all $k$, it follows that $Y_{1}$ is odd. By (2.3), it follows that $Y_{k}$ is even for all even $k$. Hence, $k_{i}$ are odd for $i=1,2,3$. Using the lemmas from Section 2, in Section 4, we get an inequality among $n_{2}$ and $k_{3}$. Then in Section 5 , we use lower bounds for linear forms in logarithms to get upper bounds on $n_{2}$ and $n_{3}$. Specifically, Lemma 5.2 shows that $n_{2} \leq 120$ and $n_{3}<10^{17}$. Thus, $Y_{k_{2}}=2^{n_{2}}-1$ for some odd $k_{2} \geq 3$ and $n_{2} \in[1,120]$. In particular, $Y_{1}$ is a divisor of $2^{n_{2}}-1$. In Section 6, we use reduction methods (the Baker-Davenport method) to reduce the upper bound on $n_{3}$ and compute all the possible candidates $\left(d, Y_{1}\right)$ such that $Y_{n}=2^{k}-1$ holds for three pairs of integers $\left(k_{i}, n_{i}\right)$ with $i=1,2,3$, $n_{1}<n_{2}<n_{3}$ and $n_{2} \leq 120$ and confirm computationally that in fact there is no such solution.

\section{An inequality among solutions to $Y_{k}=2^{n}-1$}

The aim of this section is to prove the following result.

Lemma 4.1. Assume $\left(k_{1}, n_{1}\right),\left(k_{2}, n_{2}\right),\left(k_{3}, n_{3}\right)$ are positive integer solutions $(k, n)$ to $Y_{k}=2^{n}-1$ with $k_{1}<k_{2}<k_{3}$. Then the inequality

$$
n_{2}<3 \log k_{3}
$$


holds.

Proof. Write

$$
Y_{k}=2^{n}-1 \quad(k, n)=\left(k_{2}, n_{2}\right) \quad \text { and } \quad(k, n)=\left(k_{3}, n_{3}\right),
$$

and subtract them to get

$$
2^{n_{2}}\left(2^{n_{3}-n_{2}}-1\right)=Y_{k_{3}}-Y_{k_{2}}=2 Y_{\left(k_{3}-k_{2}\right) / 2} X_{\left(k_{3}+k_{2}\right) / 2},
$$

by (2.4). Thus,

$$
n_{2}=\nu_{2}\left(2 Y_{\left(k_{3}-k_{2}\right) / 2} X_{\left(k_{3}+k_{2}\right) / 2}\right) .
$$

Since $Y_{1}$ is odd, the number $e$ in Lemma 2.1 equals 2. Thus, by Lemma 2.1, we get

$$
\begin{aligned}
n_{2} & =1+\nu_{2}\left(Y_{\left(k_{3}-k_{2}\right) / 2}\right)+\nu_{2}\left(X_{\left(k_{3}+k_{2}\right) / 2}\right) \\
& \leq 1+\nu_{2}\left(Y_{2}\right)+\nu_{2}\left(\left(k_{3}-k_{2}\right) / 4\right)+\nu_{2}\left(X_{1}\right) \\
& =2 \nu_{2}\left(X_{1}\right)+\nu_{2}\left(k_{3}-k_{2}\right) \\
& <2 \nu_{2}\left(X_{1}\right)+\frac{\log k_{3}}{\log 2},
\end{aligned}
$$

provided that $\left(k_{3}-k_{2}\right) / 2$ is even. In case $\left(k_{3}-k_{2}\right) / 2$ is odd, the last righthand side above can be replaced by $1+\nu_{2}\left(X_{1}\right)<2 \nu_{2}\left(X_{1}\right)+\log k_{3} / \log 2$, where the last inequality holds because $k_{3} \geq 3>2$. Thus, (4.1) holds regardless of the parity of $\left(k_{3}-k_{2}\right) / 2$. Now

$$
2^{n_{2}}>2^{n_{2}}-1=Y_{k_{2}}>\alpha^{k_{2}-1}
$$

where we used (2.2) for the right-most inequality, so

$$
n_{2}>\left(k_{2}-1\right)\left(\frac{\log \alpha}{\log 2}\right) \text {. }
$$

Assume first that $k_{2} \geq 5$. Then,

$$
n_{2}>4\left(\frac{\log \alpha}{\log 2}\right)>\frac{4 \log X_{1}}{\log 2} .
$$

Hence,

$$
\frac{4 \log X_{1}}{\log 2}<n_{2}<2 \nu_{2}\left(X_{1}\right)+\frac{\log k_{3}}{\log 2}<\frac{2 \log X_{1}}{\log 2}+\frac{\log k_{3}}{\log 2},
$$

which yields

$$
\log X_{1}<0.5 \log k_{3} .
$$

Thus, in this case

$$
n_{2}<2 \nu_{2}\left(X_{1}\right)+\frac{\log k_{3}}{\log 2}<\frac{2 \log X_{1}}{\log 2}+\frac{\log k_{3}}{\log 2}<\frac{2 \log k_{3}}{\log 2}<3 \log k_{3} .
$$

Assume next that $k_{2}=3$ and let $a:=\nu_{2}\left(X_{1}\right)$. Then

$$
2^{n_{2}}=Y_{3}+1=4 X_{1}^{2} Y_{1}-\left(Y_{1}-1\right) \text {. }
$$


Assume next that $Y_{1}>1$. Then the above equation shows that the three numbers $\nu_{2}\left(2^{n_{2}}\right), \nu_{2}\left(4 X_{1}^{2} Y_{1}\right)$ and $\nu_{2}\left(Y_{1}-1\right)$ cannot be distinct. Suppose that

$$
\nu_{2}\left(Y_{1}-1\right)=\nu_{2}\left(4 X_{1}^{2} Y_{1}\right)=2 a+2 .
$$

Assume $2^{a}>\sqrt{X_{1}}$. Then $2^{2 a+2}>4 X_{1}>X_{1}$ and $2^{2 a+2} \mid Y_{1}-1$. Hence, $Y_{1} \geq 1+2^{2 a+2}>X_{1}$, so

$$
1=X_{1}^{2}-d Y_{1}^{2}<X_{1}^{2}-d X_{1}^{2}=-(d-1) X_{1}^{2},
$$

a contradiction. Thus, $2^{a}<\sqrt{X_{1}}$. Next suppose that $\nu_{2}\left(4 X_{1}^{2} Y_{1}\right)=\nu_{2}\left(2^{n_{2}}\right)$. It then follows that

$$
\nu_{2}\left(Y_{1}-1\right)>\nu_{2}\left(4 X_{1}^{2} Y_{1}\right)=2 a+2,
$$

and the above argument shows again that $2^{a}<\sqrt{X_{1}}$. Finally, assume that $\nu_{2}\left(Y_{1}-1\right)=\nu_{2}\left(2^{n_{2}}\right)$. In this case, we have that $Y_{1}-1 \geq 2^{n_{2}}$. However, then

$$
2^{n_{2}}=4 X_{1}^{2} Y_{1}-\left(Y_{1}-1\right)>\left(4 X_{1}^{2}-1\right)\left(Y_{1}-1\right) \geq 2^{n_{2}}\left(4 X_{1}^{2}-1\right)>3 \cdot 2^{n_{2}},
$$

a contradiction. Thus, in all instances when $k_{2}=3$ and $Y_{1}>1$ we must have $2^{a}<\sqrt{X_{1}}$. We then have

$$
\begin{aligned}
\frac{2 \log X_{1}}{\log 2} & <\left(k_{2}-1\right) \frac{\log \alpha}{\log 2}<n_{2}<2 \nu_{2}\left(X_{1}\right)+\frac{\log k_{3}}{\log 2} \\
& =2 a+\frac{\log k_{3}}{\log 2}<\frac{\log X_{1}}{\log 2}+\frac{\log k_{3}}{\log 2},
\end{aligned}
$$

showing that $X_{1}<k_{3}$. So,

$$
n_{2}<2 a+\frac{\log k_{3}}{\log 2}<\frac{\log X_{1}}{\log 2}+\frac{\log k_{3}}{\log 2}<\frac{2 \log k_{3}}{\log 2}<3 \log k_{3} .
$$

It remains to consider the case $k_{2}=3$ and $Y_{1}=1$. This leads to

$$
2^{n_{3}}=Y_{k_{3}}+1=Y_{k_{3}}+Y_{1}=2 Y_{\left(k_{3}+1\right) / 2} X_{\left(k_{3}-1\right) / 2}
$$

(see (2.4)). Since $k_{3}>3$, it follows that $\left(k_{3}-1\right) / 2>1$. If $\left(k_{3}-1\right) / 2$ is even, then $X_{\left(k_{3}-k_{1}\right) / 2}$ is an odd integer larger than 1 (see (2.3)), which cannot divide $2^{n_{2}}$, whereas if $\left(k_{3}-k_{1}\right) / 2$ is odd, then $X_{\left(k_{3}-k_{1}\right) / 2} / X_{1}$ is an odd integer larger than 1 (see (i) of Lemma 2.1), which cannot divide $2^{n_{2}}$, either. This contradiction shows that this last case $\left(k_{2}, Y_{1}\right)=(3,1)$ is not possible, so the conclusion of the lemma follows.

\section{UPPER BOUNDS FOR $n_{2}$ AND $n_{3}$}

Using the Binet formula (2.1) of $Y_{k}$, we rewrite the equation $Y_{k}=2^{n}-1$ as

$$
Y_{k}=\frac{\alpha^{k}-\alpha^{-k}}{2 \sqrt{d}}=2^{n}-1 .
$$


This yields

$$
\left|\frac{\alpha^{k}}{2 \sqrt{d}}-2^{n}\right|=\left|\frac{\alpha^{-k}}{2 \sqrt{d}}-1\right|<2 .
$$

Dividing both sides of the inequality (5.1) by $2^{n}$, we obtain

$$
\left|\alpha^{k} 2^{-n}(2 \sqrt{d})^{-1}-1\right|<\frac{1}{2^{n-1}}
$$

We define

$$
\Lambda:=\alpha^{k} 2^{-n}(2 \sqrt{d})^{-1}-1 .
$$

Clearly, $\Lambda$ is nonzero since $\alpha^{2 k}$ is irrational. We find a lower bound on its absolute value using a result of Matveev ([12]), which we now state.

TheOREM 5.1. Let $\gamma_{1}, \ldots, \gamma_{s}$ be a real algebraic numbers and let $b_{1}, \ldots, b_{s}$ be nonzero integers. Let $D$ be the degree of the number field $\mathbb{Q}\left(\gamma_{1}, \ldots, \gamma_{s}\right)$ and let $A_{j}$ be positive real numbers satisfying

$$
A_{j}=\max \left\{D h\left(\gamma_{j}\right),\left|\log \gamma_{j}\right|, 0.16\right\} \quad \text { for } \quad j=1, \ldots, s,
$$

where for an algebraic number $\gamma$ we use $h(\gamma)$ for its usual logarithmic height. Assume that

$$
B \geq \max \left\{\left|b_{1}\right|, \ldots,\left|b_{s}\right|\right\} .
$$

If $\gamma_{1}^{b_{1}} \cdots \gamma_{s}^{b_{s}}-1 \neq 0$, then

$\left|\gamma_{1}^{b_{1}} \cdots \gamma_{s}^{b_{s}}-1\right| \geq \exp \left(-1.4 \cdot 30^{s+3} \cdot s^{4.5} \cdot D^{2}(1+\log D)(1+\log B) A_{1} \cdots A_{s}\right)$.

For us, we take $s=3, \gamma_{1}=\alpha, \gamma_{2}=2, \gamma_{3}=2 \sqrt{d}, b_{1}=k, b_{2}=-n$ and $b_{3}=-1$. The degree $D$ of the field $\mathbb{L}=\mathbb{Q}(\sqrt{d})$ containing $\alpha$ and $\sqrt{d}$ is 2 . We can take $B=n$ (since $n \geq k$ by (4.2) and the fact that $\alpha \geq 1+\sqrt{2}>2$ ). We can also take

$$
A_{1}=\log \alpha=2 h\left(\gamma_{1}\right), \quad A_{2}=2 \log 2=2 h\left(\gamma_{2}\right),
$$

and

$$
A_{3}=2 \log \alpha>2 \log (2 \sqrt{d})=2 h\left(\gamma_{3}\right) .
$$

Hence, we get that

$$
\begin{aligned}
& |\Lambda| \geq \exp \left(-1.4 \cdot 30^{6} \cdot 3^{4.5} \cdot 2^{2}(1+\log 2)(1+\log n)(\log \alpha)(2 \log 2)(2 \log \alpha)\right) \\
& \quad \geq \exp \left(-2.7 \cdot 10^{12}(1+\log n)(\log \alpha)^{2}\right) .
\end{aligned}
$$

Taking logarithms in (5.2) and comparing the resulting inequality with (5.3), we obtain

$$
n-1 \leq 3.9 \cdot 10^{12}(1+\log n)(\log \alpha)^{2} .
$$


By inequality (4.2), the fact that $k_{2} \geq 3$, Lemma 4.1, and the fact that $k_{3} \leq n_{3}$, we have that

$$
\log \alpha<\frac{n_{2} \log 2}{k_{2}-1} \leq \frac{n_{2} \log 2}{2}<\left(\frac{3 \log 2}{2}\right) \log k_{3}<1.04 \log n_{3} .
$$

Inserting the above inequality into (5.4) for $n:=n_{3}$, we get

$$
n_{3}-1 \leq 4.3 \cdot 10^{12}\left(1+\log n_{3}\right)\left(\log n_{3}\right)^{2},
$$

which gives $n_{3}<3 \times 10^{17}$. Using Lemma 4.1 , we get

$$
n_{2}<3 \log n_{3}<121 \text {. }
$$

Thus, we have the following result.

Lemma 5.2. Assume $\left(k_{1}, n_{1}\right),\left(k_{2}, n_{2}\right),\left(k_{3}, n_{3}\right)$ are positive integer solutions $(k, n)$ to $Y_{k}=2^{n}-1$ with $k_{1}<k_{2}<k_{3}$. Then

$$
n_{2} \leq 120 \quad \text { and } \quad n_{3}<3 \times 10^{17} \text {. }
$$

\section{REDUCing the BOUndS AND Finishing OFF the COMPUTATIONS}

We use the following reduction lemma originally due to Baker and Davenport ([1]).

Lemma 6.1. Let $\kappa$ be an irrational number, $M$ be a positive integer, and $p / q$ be a convergent of the continued fraction of $\kappa$ such that $q>6 M$. Let $A, B, \mu$ be some real numbers with $A>0$ and $B>1$. Let $\epsilon:=\|\mu q\|-M\|\kappa q\|$, where $\|\cdot\|$ denotes the distance from the nearest integer. If $\epsilon>0$, then there is no solution of the inequality

$$
0<|r \kappa-s+\mu|<A B^{-w}
$$

in positive integers $r, s$ and $w$ with $r \leq M$ and $w \geq \log (A q / \epsilon) / \log B$.

From now on, we distinguish two cases depending on the size of $d$.

6.1. The case $d \leq 1000$. There are 969 numbers $d \in[2,1000]$ which are not squares. We eliminate the ones for which $Y_{1}$ is even since they are not convenient for our problem. Note that these include the ones for which the period of the continued fraction of $\sqrt{d}$ has odd length since for those ones the equation $U^{2}-d V^{2}=-1$ has infinitely many positive integer solutions $(U, V)$ and if $\left(U_{1}, V_{1}\right)$ is the smallest one, then $Y_{1}=2 U_{1} V_{1}$ is even. There are 282 values of $d$ remaining to deal with and they include the powers of 2 of odd exponent larger than 1 in this range, namely 8, 32, 128, 512 .

We turn to (5.2) for $n:=n_{3}(\geq 3)$ and using the fact that the inequality $\left|e^{x}-1\right|<y$ for real $x$ and $y \in(0,1 / 2)$ implies $|x|<2 y$, we deduce that it yields

$$
|k \log \alpha-(n+1) \log 2-\log \sqrt{d}|<\frac{1}{2^{n-2}} \quad \text { for } \quad(k, n)=\left(k_{3}, n_{3}\right),
$$


which in turn leads to

$$
\left|k\left(\frac{\log \alpha}{\log 2}\right)-(n+1)+\left(\frac{-\log (\sqrt{d})}{\log 2}\right)\right|<\frac{0.25(\log 2)^{-1}}{2^{n}}<\frac{0.37}{2^{n}} .
$$

So, we apply Lemma 6.1 with the data

$$
\kappa:=\frac{\log \alpha}{\log 2}, \quad \mu:=\frac{-\log (\sqrt{d})}{\log 2}, \quad A:=0.37, \quad B:=2 .
$$

We take $M:=3 \times 10^{17}$ and use the fact that $k<n<M$. If $F_{m}$ is the $m$ th Fibonacci number, then $F_{98}>1.3 \times 10^{20}>6 \mathrm{M}$, which shows that $q_{98}>6 \mathrm{M}$. So, we choose $p / q$ to be $p_{98} / q_{98}$. We apply the Baker-Davenport reduction for all our values of $d$ except for $d \in\{8,512\}$. We get that $\epsilon>0.000407$ in all instances and $q<1.6 \times 10^{62}$. This gives

$$
n<\log \left(0.37 \times 1.6 \times 10^{62} / 0.0004\right) / \log 2<206 .
$$

Hence, $n_{3} \leq 205$. When $d \in\{8,512\}, q_{98}$ is even so $\left\|q_{98} \mu\right\|=0$, showing that $\epsilon<0$. In this case, we use $q_{97}$ instead which is odd, and which works and gives the same conclusion because

$$
\epsilon=\left\|q_{97} \mu\right\|-M\left\|q_{97} \tau\right\| \geq 0.5-\frac{M}{q_{98}} \geq 0.5-\frac{3 \times 10^{17}}{F_{98}}>0.5-0.003>0.4 .
$$

Thus, for each of our values of $d$, we computed $\left\{Y_{k}\right\}_{k \geq 1}$ for all $k \leq 205$ using the recurrence relation $Y_{k+2}=2 X_{1} Y_{k+1}-Y_{k}$ for all $k \geq 0$ with $Y_{0}=0, Y_{1}=1$ and counted in each case how many odd $k$ are there in $[1,205]$ such that $Y_{k}+1$ is a power of 2 . No example with 3 such $k$ was found. This deals with the case $d \leq 1000$.

6.2. The case $d>1000$. Here, we note first that since $\alpha>2 \sqrt{d} \geq 2 \sqrt{1001}$ and $k_{2} \geq 3$, inequality (4.2) gives

$$
n_{2}>\left(k_{2}-1\right)\left(\frac{\log \alpha}{\log 2}\right)>\frac{2 \log (2 \sqrt{1001})}{\log 2}>11.9,
$$

so $n_{2} \in[12,120]$. We use the fact that

$$
Y_{k}=\frac{\alpha^{k}-\beta^{k}}{2 \sqrt{d}}=Y_{1}\left(\frac{\left(X_{1}+\sqrt{X_{1}^{2}-1}\right)^{k}-\left(X_{1}-\sqrt{X_{1}^{2}-1}\right)^{k}}{2 \sqrt{X_{1}^{2}-1}}\right)=Y_{1} P_{k}\left(X_{1}\right),
$$

where

$$
\begin{aligned}
P_{k}\left(X_{1}\right) & =\frac{\left(X_{1}+\sqrt{X_{1}^{2}-1}\right)^{k}-\left(X_{1}-\sqrt{X_{1}^{2}-1}\right)^{k}}{2 \sqrt{X_{1}^{2}-1}} \\
& =\sum_{\substack{0 \leq i \leq k \\
i \equiv k-1}}\left(\begin{array}{c}
k \\
i
\end{array}\right) X_{1}^{i}\left(X_{1}^{2}-1\right)^{(k-1-i) / 2}
\end{aligned}
$$


is in $\mathbb{Z}\left[X_{1}\right]$. We fix $n_{2} \in[12,120]$ and let $Y_{1}$ be a divisor of $2^{n_{2}}-1$. Later we will see that $Y_{1}<2^{n_{2} / 3}$. Then $\alpha=X_{1}+\sqrt{d} Y_{1}>2 Y_{1} \sqrt{1001}$. Hence,

$$
k_{2}-1 \leq \frac{n_{2} \log 2}{\log \alpha}<\frac{120 \log 2}{\log \left(2 Y_{1} \sqrt{1001}\right)} .
$$

Since $Y_{1} \geq 1$, we have that $k_{2} \leq 21$. Now

$$
P_{k_{2}}\left(X_{1}\right)=\frac{2^{n_{2}}-1}{Y_{1}}
$$

The following lemma gives the exact value of $X_{1}$.

LEMMA 6.2. In (6.3), we have

$$
X_{1}=\left\lfloor 0.5\left(\frac{2^{n_{2}}-1}{Y_{1}}\right)^{1 /\left(k_{2}-1\right)}+0.5\right\rfloor .
$$

Proof. The sequence $\left\{P_{k}\left(X_{1}\right)\right\}_{k \geq 0}$ satisfies the recurrence

$$
P_{k+2}\left(X_{1}\right)=\left(2 X_{1}\right) P_{k+1}\left(X_{1}\right)-P_{k}\left(X_{1}\right) \quad \text { for all } \quad k \geq 0,
$$

with $P_{0}\left(X_{1}\right)=0, P_{1}\left(X_{1}\right)=1$. Thus, $P_{2}\left(X_{1}\right)=2 X_{1}$. It follows by induction using recurrence formula (6.5) that $P_{k}\left(X_{1}\right) \leq\left(2 X_{1}\right)^{k-1}$ holds for all $k \geq 1$ and the inequality is strict for $k \geq 2$. Next note that for $k$ odd and integer $X_{1}$, we have that

$$
\begin{aligned}
P_{k}\left(X_{1}\right) & \geq \frac{\left(X_{1}+\sqrt{X_{1}^{2}-1}\right)^{k}-1}{2 \sqrt{X_{1}^{2}-1}} \\
& >\left(2 \sqrt{X_{1}^{2}-1}\right)^{k-1}-\frac{1}{2 \sqrt{X_{1}^{2}-1}} \\
& \geq\left(4 X_{1}^{2}-4\right)^{(k-1) / 2}-\frac{1}{2 \sqrt{1001}},
\end{aligned}
$$

so

$$
P_{k_{2}}\left(X_{1}\right) \geq\left(4 X_{1}^{2}-4\right)^{\left(k_{2}-1\right) / 2}
$$

Thus, we get that

$$
\left(4 X_{1}^{2}-4\right)^{\left(k_{2}-1\right) / 2} \leq P_{k_{2}}\left(X_{1}\right)=\frac{2^{n_{2}}-1}{Y_{1}}<\left(2 X_{1}\right)^{k_{2}-1}=\left(4 X_{1}^{2}\right)^{\left(k_{2}-1\right) / 2},
$$

so

$$
4 X_{1}^{2}-4<\left(\frac{2^{n_{2}}-1}{Y_{1}}\right)^{2 /\left(k_{2}-1\right)}<4 X_{1}^{2}
$$


We thus get that

$$
\begin{aligned}
\left(\frac{2^{n_{2}}-1}{Y_{1}}\right)^{1 /\left(k_{2}-1\right)} & <2 X_{1}<\sqrt{\left(\frac{2^{n_{2}}-1}{Y_{1}}\right)^{2 /\left(k_{2}-1\right)}+4} \\
& =\left(\frac{2^{n_{2}}-1}{Y_{1}}\right)^{1 /\left(k_{2}-1\right)}\left(1+\frac{4}{\left(\frac{2^{n_{2}}-1}{Y_{1}}\right)^{2 /\left(k_{2}-1\right)}}\right)^{1 / 2} .
\end{aligned}
$$

In the right-hand side above, we apply Bernoulli's inequality

$$
(1+a)^{b}<1+a b \quad \text { valid for } \quad a, b \in(0,1),
$$

with $\left.a=4 /\left(2^{n_{2}}-1\right) / Y_{1}\right)^{2 /\left(k_{2}-1\right)}, b=1 / 2$. We need to check that $a \in(0,1)$. But if this were not so, then $\left.\left(2^{n_{2}}-1\right) / Y_{1}\right)^{2 /\left(k_{2}-1\right)} \leq 4$ and (6.6) would lead to the conclusion that $2 X_{1} \leq \sqrt{8}$, so $X_{1}=1$, a contradiction. Thus, we can apply Bernoulli's inequality and get that

$$
\left(1+\frac{4}{\left(\frac{2^{n_{2}-1}}{Y_{1}}\right)^{2 /\left(k_{2}-1\right)}}\right)^{1 / 2}<1+\frac{2}{\left(\frac{2^{n_{2}-1}}{Y_{1}}\right)^{2 /\left(k_{2}-1\right)}} .
$$

Thus,

$$
\begin{aligned}
2 X_{1} & <\left(\frac{2^{n_{2}}-1}{Y_{1}}\right)^{1 /\left(k_{2}-1\right)}\left(1+\frac{2}{\left(\frac{2^{n_{2}}-1}{Y_{1}}\right)^{2 /\left(k_{2}-1\right)}}\right) \\
& <\left(\frac{2^{n_{2}}-1}{Y_{1}}\right)^{1 /\left(k_{2}-1\right)}+\frac{2}{\left(\frac{2^{n_{2}-1}}{Y_{1}}\right)^{1 /\left(k_{2}-1\right)}} \\
& <\left(\frac{2^{n_{2}}-1}{Y_{1}}\right)^{1 /\left(k_{2}-1\right)}+1 .
\end{aligned}
$$

We thus get that

$$
0.5\left(\frac{2^{n_{2}}-1}{Y_{1}}\right)^{1 /\left(k_{2}-1\right)}<X_{1}<0.5\left(\frac{2^{n_{2}}-1}{Y_{1}}\right)^{1 /\left(k_{2}-1\right)}+0.5
$$

and $X_{1}$ is an integer. So, $X_{1}$ is indeed given by formula (6.4).

We can now justify that $Y_{1}<2^{n_{2} / 3}$. Indeed, we have $X_{1}^{2}>Y_{1}^{2}$, so $X_{1} \geq Y_{1}+1$. Thus,

$$
Y_{1}+1 \leq X_{1}<0.5\left(\frac{2^{n_{2}}-1}{Y_{1}}\right)^{1 /\left(k_{2}-1\right)}+0.5
$$


showing that

$$
Y_{1}<0.5\left(\frac{2^{n_{2}}-1}{Y_{1}}\right)^{1 /\left(k_{2}-1\right)}<\left(\frac{2^{n_{2}}}{Y_{1}}\right)^{1 / 2}
$$

giving $Y_{1}^{3}<2^{n_{2}}$, so $Y_{1}<2^{n_{2} / 3}$.

We now describe the computations. We generated the list of pairs $\left(n_{2}, Y_{1}\right)$ with $n_{2} \in[12,120]$ and $Y_{1}$ a divisor of $2^{n_{2}}-1$ satisfying $Y_{1}<2^{n_{2} / 3}$. There are 27401 such pairs. For each odd $k_{2} \geq 3$ satisfying (6.2), we computed $X_{1}$ via formula (6.4). This is of course only a candidate. To check that it is indeed a solution we checked whether with this value of $X_{1}$, the formula

$$
P_{k_{2}}\left(X_{1}\right)=\sum_{j=0}^{\left(k_{2}-1\right) / 2}\left(\begin{array}{l}
k_{2} \\
2 j
\end{array}\right) X_{1}^{2 j}\left(X_{1}^{2}-1\right)^{\left(k_{2}-1\right) / 2-j}=\frac{2^{n_{2}}-1}{Y_{1}}
$$

holds. If it did, we further checked that $Y_{1}^{2} \mid X_{1}^{2}-1$ and that $d:=\left(X_{1}^{2}-\right.$ $1) / Y_{1}^{2}>1000$. If all these tests are satisfied we asked our code to return a vector of 5 components namely $\left(d, X_{1}, Y_{1}, k_{2}, n_{2}\right)$. Over the 27401 such pairs we obtained only 55 vectors. They all had $Y_{1}=1, k_{2}=3$ and $d=2^{2 \ell}-1$, for $\ell \in[5,59]$. Thus, these are exactly the parametric family mentioned after the statement of the main theorem which are in the tested range so by the argument from the end of the proof of Lemma 4.1 there cannot be a third solution $\left(k_{3}, n_{3}\right)$. Just for the fun of it, we returned to (6.1) and we did the Baker-Davenport reduction over all the 55 values of $d$ with the same $M$ and $q=q_{98}$. The smallest $\epsilon$ obtained was larger than 0.015 and all $q$ satisfied $q<1.9 \times 10^{75}$. This gives $n_{3} \leq 255$. Since $k_{3} \leq n_{3}$, we get $k_{3} \leq 255$. Now we generated, for each of the 55 values of $d$, the numbers $Y_{k}$ for $k$ odd in [5, 255] and tested whether $Y_{k}+1$ is a power of 2 . No additional example was found. The theorem is therefore proved.

All calculations were done with Mathematica.

\section{ACKNowledgements}

The authors thank the anonymous referees for a careful reading of the manuscript and useful suggestions and Professor Mouhamed Moustapha Fall for useful discussions. This work started during a very enjoyable visit of F. L. at AIMS Sénégal in February 2018 and ended during a research stay of F. L. at the Max Planck Institute for Mathematics in Bonn between September 1, 2019 and February 29, 2020. F. L. thanks these institutions for the hospitality and support. In addition, F. L. was supported in parts by Grant CPRR160325161141 of NRF and the Number Theory Focus Area Grant of CoEMaSS at Wits (South Africa).

\section{REFERENCES}

[1] A. Baker and H. Davenport, The equations $3 x^{2}-2=y^{2}$ and $8 x^{2}-7=z^{2}$, Q. J. Math. 20 (1969), 129-137. 
[2] M. A. Bennett, On the number of solutions of simultaneous Pell equations, J. Reine Angew. Math. 498 (1998), 173-199.

[3] J. H. E. Cohn, The Diophantine equation $x^{4}-D y^{2}=1$, II, Acta Arith. 78 (1997), 401-403.

[4] A. Dossavi-Yovo, F. Luca and A. Togbé, On the $x$-coordinates of Pell equations which are rep-digits, Publ. Math. Debrecen 88 (2016), 381-399.

[5] H. S. Erazo, C. A. Gómez and F. Luca, On Pillai's problem with X-coordinates of Pell equations and powers of 2, J. Number Theory 203 (2019), 294-309.

[6] B. Faye and F. Luca, On the X-coordinates of Pell equations which are rep-digits, Fibonacci Quart. 56 (2018), 52-62.

[7] S. Laisharam, F. Luca and M. Sias, On the Diophantine equation $n !^{2} \pm 1=d v^{2}$, preprint, 2018.

[8] W. Ljunggren, Einige Eigenshaften der Einheiten reeller quadratischer und reinbiquadratischer Zahl Körper auf die Lösung einer Klasse unbestimmer Gleichungen 4. Grades, Skr. Norske Vid.-Akad. Oslo I Mat.-Naturv. Klasse 12 (1936), 73 pp.

[9] F. Luca, A. Montejano, L. Szalay and A. Togbé, On the X-coordinates of Pell equations which are Tribonacci numbers, Acta Arith. 179 (2017), 25-35.

[10] F. Luca and A. Togbé, On the x-coordinates of Pell equations which are Fibonacci numbers, Math. Scand. 122 (2018), 18-30.

[11] D.W. Masser and J.H. Rickert, Simultaneous Pell equations, J. Number Theory 61 (1996), 52-66.

[12] E. M. Matveev, An explicit lower bound for a homogeneous rational linear form in the logarithms of algebraic numbers, II, Izv. Math. 64 (2000), 1217-1269.

B. Faye-Fall

UFR SAT

Université Gaston Berger

Saint-Louis 32002

Sénégal

E-mail: bernadette.fayee@gmail.com

F. Luca

School of Mathematics, University of the Witwatersrand

Private Bag X3, Wits 2050, Johannesburg

South Africa

and

Research Group in Algebraic Structures and Applications

King Abdulaziz University, Jeddah

Saudi Arabia

and

Centro de Ciencias Matemáticas, UNAM

Morelia

Mexico

E-mail: florian.luca@wits.ac.za

Received: 19.8.2019.

Revised: $17.10 .2019 . \quad \& 30.11 .2019$. 